\title{
Exact Robust Solution to TW-ToA-Based Target Localization Problem With Clock Imperfections
}

\author{
Slavisa Tomic ${ }^{(0)}$ and Marko Beko
}

\begin{abstract}
This letter addresses the problem of target localization based on two-way time of arrival (TW-ToA) measurements with clock imperfections. In addition to the target location, the turn-around times and clock skews are considered unknown. Since an optimal estimator for this problem cannot be tackled directly, we approximate it by a suboptimal, robust one, formulated as a generalized trust region subproblem. Even though nonconvex in general, exact solution of the derived estimator can be obtained by just a bisection procedure. Simulation results validate the effectiveness of the proposed technique, matching the performance of the state of the art with significantly lower computational complexity.
\end{abstract}

Index Terms-Clock imperfections, generalized trust region subproblem (GTRS), robust localization, two-way time of arrival (TW-ToA).

\section{INTRODUCTION}

$\mathbf{R}$ ANGE-BASED localization has attracted much attention in the research society recently [1]-[10]. Range measurements can be extracted using various schemes, and one of them is from two-way time of arrival (TW-ToA) measurements, in which target records time stamps to compute the measurements [11]-[16]. Albeit such a technique is robust to clock offsets, TW-ToA-based methods suffer from imperfect clock skews (CSs) and unknown processing times called turn-around times (TATs).

A number of TW-ToA-based localization techniques can be found in this literature. A linear least squares (LLSs) estimator was proposed in [11] to jointly estimate the CS and the target location, where the TATs were estimated by a loop back test. In [12], a Euclidean distance matrix (EDM) based approach was described for cooperative localization with joint estimation of

Manuscript received October 2, 2017; revised January 22, 2018; accepted February 26, 2018. Date of publication February 28, 2018; date of current version March 14, 2018. This work was supported in part by Fundação para a Ciência e a Tecnologia under Project PEst-OE/EEI/UI0066/2014 and Project UID/EEA/50009/2013; and in part by the Program Investigador Fundação para a Ciência e Tecnologia under Grant IF/00325/2015. The associate editor coordinating the review of this manuscript and approving it for publication was Prof. Parv Venkitasubramaniam. (Corresponding author: Slavisa Tomic.)

$\mathrm{S}$. Tomic is with the COPELABS, Universidade Lusófona de Humanidades e Tecnologias, Lisboa 1749-024, Portugal, and also with the Institute for Systems and Robotics/Instituto Superior Tecnico, Laboratory for Robotics and Engineering Systems, Universidade de Lisboa, Lisbon 1049-001, Portugal (e-mail: slavisa.tomic@ulusofona.pt).

M. Beko is with the COPELABS, Universidade Lusófona de Humanidades e Tecnologias, Lisboa 1749-024, Portugal, and also with the Centre of Technology and Systems/Instituto de Desenvolvimento de Novas Tecnologias, Campus da Faculdade de Ciências e Tecnologia/Universidade NOVA de Lisboa, Lisboa 1099-085, Portugal (e-mail: beko.marko@ulusofona.pt).

Color versions of one or more of the figures in this letter are available online at http://ieeexplore.ieee.org.

Digital Object Identifier 10.1109/LSP.2018.2810829 the TATs and the target location. A semidefinite cone programming (SDP) estimator for asynchronous time of arrival target localization was proposed in [14]. Gholami et al. [15] propose a concave-convex procedure (CCP) based on second-order cone programming (SOCP), and an extended generalized trust region (EGTR) subproblem to solve the TW-ToA localization problem with clock imperfections. A robust SOCP (R-SOCP) estimator, where the authors treated the CSs and the TATs as nuisance parameters, was described in [16].

Most of the above techniques are too expensive in the sense of computational cost, and the other ones are not as accurate. Therefore, here, we develop a novel estimator that represents an excellent tradeoff between accuracy and computational cost. Under the assumption that the ranges of the TATs and CSs can be estimated (which is fairly simple in the calibration phase [16]), we convert the originally nonconvex problem into a generalized trust region subproblem (GTRS) framework by applying robust squared-range (R-SR) and weighted least squares (WLS) criterion. The solution to the derived estimator is readily obtained by a bisection procedure [17]. Based on an iterative scheme, we partially mitigate the influence of the CSs and the TATs, by calculating their maximum likelihood (ML) estimates and we use them to further enhance the accuracy of the location estimate. Unlike most of the above described algorithms, computational complexity of the new one is linear in the number of reference points (anchors), and its performance matches that of the state of the art (SoA).

\section{PROBLEM FORMULATION}

Denote by $\boldsymbol{x}, \boldsymbol{a}_{i} \in \mathbb{R}^{k}$, the unknown location of the target and the known location of the $i$ th anchor, $i=1, \ldots, N$, respectively, in a $k$-dimensional wireless sensor network $(k=1,2,3)$. The target estimates the ranges to the anchors according to a TWToA ranging protocol [11], [15], [16], i.e., the target sends a packet to the anchors, they process it, and return the packet to the target. This processing time at the anchors is known as the TAT, $T_{i}$, and is unknown in general. The TW-ToA measurements are modeled as [11], [15], [16]

$$
t_{i}=\omega_{0}\left(\frac{\left\|\boldsymbol{x}-\boldsymbol{a}_{i}\right\|}{c}+\frac{T_{i}}{2}\right)+\frac{\tilde{n}_{i}}{2}, \text { for } i=1, \ldots, N
$$

where $\omega_{0}$ is the target's CS, $c$ is the propagation speed, and $\tilde{n}_{i} \sim \mathcal{N}\left(\tilde{\mu}_{i}, \tilde{\sigma}_{i}^{2}\right)$ is a random (positive) delay introduced by the target during packet interception, modeled as a positive-mean Gaussian distribution [18].

By multiplying with $c$ and subtracting $c \tilde{\mu} / 2$ from both sides of (1), the following range measurement model is obtained:

$$
d_{i}=\omega_{0}\left(\left\|\boldsymbol{x}-\boldsymbol{a}_{i}\right\|+\frac{d_{T_{i}}}{2}\right)+\frac{n_{i}}{2}
$$


where $d_{i}=c t_{i}-c \tilde{\mu}_{i} / 2, d_{T_{i}}=c T_{i}$, and $n_{i}=c\left(\tilde{n}_{i}-\tilde{\mu}_{i}\right)$, i.e., $n_{i} \sim \mathcal{N}\left(0, \sigma_{i}^{2}\right)$, with $\sigma_{i}=c \tilde{\sigma}_{i}$.

Based on (2), the ML estimator [19] of $\boldsymbol{x}, \omega_{0}$ and $d_{T i}(i=$ $1, \ldots, N)$ can be formulated as

$$
\left[\widehat{\boldsymbol{x}}^{T}, \widehat{\omega}_{0}, \widehat{d}_{T_{i}}\right]^{T}=\underset{\left[\boldsymbol{x}^{T}, \omega_{0}, d_{T_{i}}\right]^{T}}{\arg \min } \sum_{i=1}^{N} \frac{\left(d_{i}-\omega_{0}\left(\left\|\boldsymbol{x}-\boldsymbol{a}_{i}\right\|+\frac{d_{T_{i}}}{2}\right)\right)^{2}}{\sigma_{i}^{2}} .
$$

The ML estimator is nonconvex with no closed-form solution; thus, (3) cannot be tackled directly. In Section III, we develop a robust estimator that is an approximation of the ML one, but whose exact solution is obtained via a bisection procedure.

\section{PROPOSED RobUST Estimator}

Besides being nonconvex, (3) is also underdetermined, since the number of unknowns $(N+k+1)$ is greater than the number of observations $(N)$. Therefore, some approximations are required to solve (3).

We begin by assuming that the ranges of $T_{i}$ can be estimated during the calibration phase [16], i.e. $T_{i} \in\left[T_{i}^{\mathrm{low}}, T_{i}^{\mathrm{upp}}\right]$ for $i=$ $1, \ldots, N$, which leads to $d_{T_{i}} \in\left[l_{i}, u_{i}\right]$, with $l_{i}=c T_{i}^{\text {low }}$ and $u_{i}=$ $c T_{i}^{\mathrm{upp}}$. Moreover, since $\omega_{0}$ varies around 1 , we can express it as $\omega_{0}=1+\delta_{0}$, where $\left|\delta_{0}\right| \leq \delta_{\max } \ll 1$ and $\delta_{\max }$ is a known constant [13], [16].

By substituting $\omega_{0}=1+\delta_{0}$ in (2), one gets

$$
d_{i}=\left\|\boldsymbol{x}-\boldsymbol{a}_{i}\right\|+\frac{d_{T_{i}}}{2}+\delta_{0}\left(\left\|\boldsymbol{x}-\boldsymbol{a}_{i}\right\|+\frac{d_{T_{i}}}{2}\right)+\frac{n_{i}}{2} .
$$

Note that for sufficiently small noise, from (2), we can write

$$
\delta_{0}\left(\left\|\boldsymbol{x}-\boldsymbol{a}_{i}\right\|+\frac{d_{T_{i}}}{2}\right)=\frac{\delta_{0}\left(d_{i}-n_{i} / 2\right)}{\omega_{0}} \approx \delta_{0} d_{i}
$$

and thus, (4) is approximated by

$$
d_{i} \approx\left\|\boldsymbol{x}-\boldsymbol{a}_{i}\right\|+\frac{d_{T_{i}}}{2}+\delta_{0} d_{i}+\frac{n_{i}}{2} .
$$

Subtracting $\left(l_{i}+u_{i}\right) / 4$ from both sides of (5), yields

$$
d_{i}-\frac{l_{i}+u_{i}}{4} \approx\left\|\boldsymbol{x}-\boldsymbol{a}_{i}\right\|+\frac{d_{T_{i}}}{2}-\frac{l_{i}+u_{i}}{4}+\delta_{0} d_{i}+\frac{n_{i}}{2}
$$

which can be rewritten as

$$
\left(\tilde{d}_{i}-e_{i}\right)^{2} \approx\left\|\boldsymbol{x}-\boldsymbol{a}_{i}\right\|^{2}+n_{i}\left\|\boldsymbol{x}-\boldsymbol{a}_{i}\right\|+\frac{n_{i}^{2}}{4}
$$

by squaring (6) and letting $\tilde{d}_{i}=d_{i}-\left(l_{i}+u_{i}\right) / 4$ and $e_{i}=$ $d_{T_{i}} / 2-\left(l_{i}+u_{i}\right) / 4+\delta_{0} d_{i}$. Furthermore, note that

$$
\begin{aligned}
& \left|e_{i}\right|=\left|\frac{d_{T_{i}}}{2}-\frac{l_{i}+u_{i}}{4}+\delta_{0} d_{i}\right| \stackrel{\text { I.1 }}{\leq}\left|\frac{d_{T_{i}}}{2}-\frac{l_{i}+u_{i}}{4}\right|+\left|\delta_{0} d_{i}\right| \\
& \stackrel{\text { I.2 }}{\leq} \frac{u_{i}-l_{i}}{4}+\delta_{\max } d_{i} \triangleq \rho_{i}
\end{aligned}
$$

where I.1 follows from the triangle inequality and I.2 follows from $l_{i} \leq d_{T_{i}} \leq u_{i}$ and $\left|\delta_{0}\right| \leq \delta_{\max }$. From (7), we can write ${ }^{1}$

$$
\frac{\left(\tilde{d}_{i}-e_{i}\right)^{2}-\left\|\boldsymbol{x}-\boldsymbol{a}_{i}\right\|^{2}}{\left\|\boldsymbol{x}-\boldsymbol{a}_{i}\right\|}=n_{i}+\frac{n_{i}^{2}}{4\left\|\boldsymbol{x}-\boldsymbol{a}_{i}\right\|} .
$$

Define $\boldsymbol{\xi}=\left[\xi_{i}\right]^{T}$, such that $\xi_{i}=\frac{\left(\tilde{d}_{i}-e_{i}\right)^{2}-\left\|\boldsymbol{x}-\boldsymbol{a}_{i}\right\|^{2}}{\left\|\boldsymbol{x}-\boldsymbol{a}_{i}\right\|}$. Then, for sufficiently low noise power, we can approximate $\xi$ by

$$
\boldsymbol{\xi} \sim \mathcal{N}\left(\mathbf{0}_{N \times 1}, \boldsymbol{\Psi}\right)
$$

${ }^{1}$ Note that by squaring (6), $n_{i}$ is not Gaussian anymore. Nonetheless, for low noise power, we can disregard the second-order noise term. with $\boldsymbol{\Psi}=\operatorname{diag}\left\{\left[\sigma_{1}^{2}, \ldots, \sigma_{N}^{2}\right]\right\}$, and $\operatorname{diag}\{\bullet\}$ denotes a square diagonal matrix. The probability density function of $\boldsymbol{\xi}$ is

$$
p(\boldsymbol{\xi})=(2 \pi)^{-\frac{N}{2}}|\boldsymbol{\Psi}|^{-\frac{1}{2}} \exp \left\{-\frac{1}{2} \boldsymbol{\xi}^{T} \boldsymbol{\Psi}^{-1} \boldsymbol{\xi}\right\}
$$

where $|\bullet|$ denotes a determinant of a matrix. Maximizing the $\log$ of (10) with respect to $\boldsymbol{x}$ for worst case $e_{i}$ gives

$$
\underset{\boldsymbol{x}}{\operatorname{minimize}} \underset{e_{i}}{\operatorname{maximize}} \sum_{i=1}^{N}\left(\frac{\left(\tilde{d}_{i}-e_{i}\right)^{2}-\left\|\boldsymbol{x}-\boldsymbol{a}_{i}\right\|^{2}}{\left\|\boldsymbol{x}-\boldsymbol{a}_{i}\right\|}\right)^{2}
$$

which can be rewritten as

$$
\underset{\boldsymbol{x}}{\operatorname{minimize}} \underset{e_{i}}{\operatorname{maximize}} \sum_{i=1}^{N} f^{2}\left(e_{i}\right)
$$

by letting $f\left(e_{i}\right)=\frac{\left|\left(\tilde{d}_{i}-e_{i}\right)^{2}-\left\|\boldsymbol{x}-\boldsymbol{a}_{i}\right\|^{2}\right|}{\left\|\boldsymbol{x}-\boldsymbol{a}_{i}\right\|}$. Notice that

$$
\underset{e_{i}}{\operatorname{maximize}} \sum_{i=1}^{N} f^{2}\left(e_{i}\right)=\sum_{i=1}^{N}\left[\underset{e_{i}}{\operatorname{maximize}} f\left(e_{i}\right)\right]^{2} .
$$

Thus, solving (12) equals finding the maximum of $f\left(e_{i}\right)$ under the condition: $\left|e_{i}\right| \leq \rho_{i}$. Hence, two cases are considered as follows:

$$
\text { 1) } \begin{array}{rl}
\left|\dot{\tilde{d}}_{i}\right| \leq \rho_{i}: \max _{e_{i}} & f\left(e_{i}\right)=\max \left\{f\left(-\rho_{i}\right), f\left(\tilde{d}_{i}\right), f\left(\rho_{i}\right)\right\} \\
f\left(-\rho_{i}\right) & =\frac{\left|\left(\tilde{d}_{i}+\rho_{i}\right)^{2}-\left\|\boldsymbol{x}-\boldsymbol{a}_{i}\right\|^{2}\right|}{\left\|\boldsymbol{x}-\boldsymbol{a}_{i}\right\|} \\
f\left(\tilde{d}_{i}\right) & =\frac{\left\|\boldsymbol{x}-\boldsymbol{a}_{i}\right\|^{2}}{\left\|\boldsymbol{x}-\boldsymbol{a}_{i}\right\|} \\
f\left(\rho_{i}\right) & =\frac{\left|\left(\tilde{d}_{i}-\rho_{i}\right)^{2}-\left\|\boldsymbol{x}-\boldsymbol{a}_{i}\right\|^{2}\right|}{\left\|\boldsymbol{x}-\boldsymbol{a}_{i}\right\|} .
\end{array}
$$

2) $\left|\tilde{d}_{i}\right|>\rho_{i}: \max _{e_{i}} f\left(e_{i}\right)=\max \left\{f\left(-\rho_{i}\right), f\left(\rho_{i}\right)\right\}$, with $f\left(-\rho_{i}\right), f\left(\rho_{i}\right)$ given by (14a) and (14c), respectively.

A similar line of reasoning was presented in [16]. However, instead of applying SOCP relaxation technique, here we take a different approach. Notice that $\max \{a, b, c\} \leq a+b+c$, for $a, b, c \geq 0$. Thus, instead of tackling (11) directly, we minimize an upper bound on (11), i.e.,

$$
\begin{aligned}
& \underset{\boldsymbol{x}}{\operatorname{minimize}} \sum_{i=1}^{N}\left(\frac{\left(\tilde{d}_{i}+\rho_{i}\right)^{2}-\left\|\boldsymbol{x}-\boldsymbol{a}_{i}\right\|^{2}}{\left\|\boldsymbol{x}-\boldsymbol{a}_{i}\right\|}\right)^{2} \\
& +\sum_{i=1}^{N}\left(\frac{\left(\tilde{d}_{i}-\rho_{i}\right)^{2}-\left\|\boldsymbol{x}-\boldsymbol{a}_{i}\right\|^{2}}{\left\|\boldsymbol{x}-\boldsymbol{a}_{i}\right\|}\right)^{2}+\sum_{\substack{i=1 \\
\text { if }\left|\tilde{d}_{i}\right| \leq \rho_{i}}}^{N}\left(\frac{\left\|\boldsymbol{x}-\boldsymbol{a}_{i}\right\|^{2}}{\left\|\boldsymbol{x}-\boldsymbol{a}_{i}\right\|}\right)^{2} .
\end{aligned}
$$

Note that both the denominator and numerator in (15) are functions of $\boldsymbol{x}$, which makes it highly nonconvex. Hence, we first introduce weights, $w_{i}=1-d_{i} / \sum_{i=1}^{N} d_{i}$, to give more importance to nearby links, and we substitute (15) with

$$
\begin{aligned}
\underset{\boldsymbol{x}}{\operatorname{minimize}} & \sum_{i=1}^{N} w_{i}\left(\frac{\left(\tilde{d}_{i}+\rho_{i}\right)^{2}-\left\|\boldsymbol{x}-\boldsymbol{a}_{i}\right\|^{2}}{\tilde{d}_{i}}\right)^{2} \\
+ & \sum_{i=1}^{N} w_{i}\left(\frac{\left(\tilde{d}_{i}-\rho_{i}\right)^{2}-\left\|\boldsymbol{x}-\boldsymbol{a}_{i}\right\|^{2}}{\tilde{d}_{i}}\right)^{2}+\sum_{\substack{i=1 \\
\text { if }\left|\tilde{d}_{i}\right| \leq \rho_{i}}}^{N} w_{i}\left(\frac{\left\|\boldsymbol{x}-\boldsymbol{a}_{i}\right\|^{2}}{\tilde{d}_{i}}\right)^{2}
\end{aligned}
$$




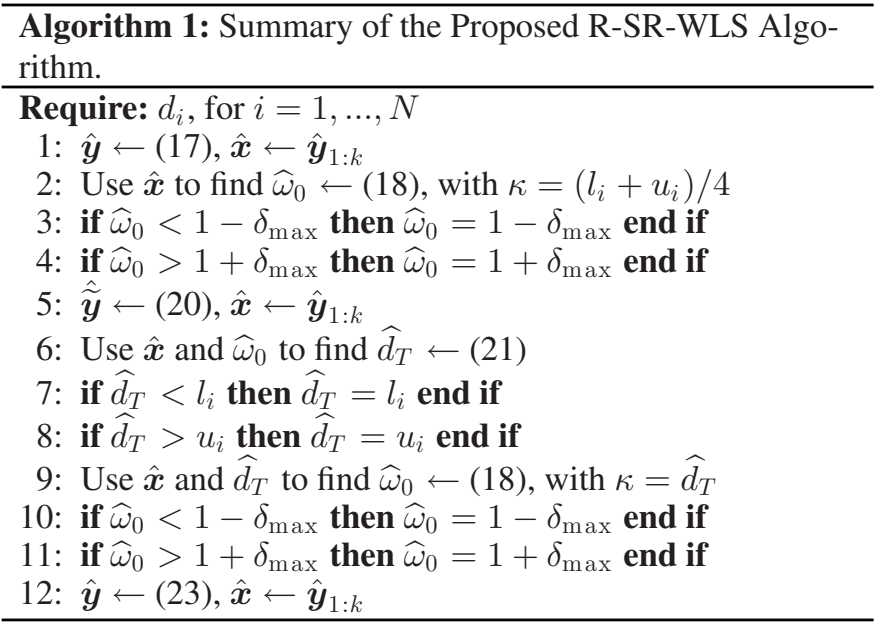

which can be reformulated as

$$
\underset{\boldsymbol{y}=\left[\boldsymbol{x}^{T},\|\boldsymbol{x}\|^{2}\right]^{T}}{\operatorname{minimize}}\left\{\left\|\boldsymbol{W}_{1}\left(\boldsymbol{A}_{1} \boldsymbol{y}-\boldsymbol{p}_{1}\right)\right\|^{2}: \boldsymbol{y}^{T} \boldsymbol{D} \boldsymbol{y}+2 \boldsymbol{g}^{T} \boldsymbol{y}=0\right\}
$$

where $\boldsymbol{W}_{1}=\operatorname{diag}\{\widetilde{\boldsymbol{w}}\}, \quad \widetilde{\boldsymbol{w}}=\left[\widetilde{w}_{i}\right]^{T}, \quad \widetilde{w}_{i}=\frac{\sqrt{w_{i}}}{\tilde{d}_{i}} \quad$ for $\quad i=$ $1, \ldots, 2 N, \widetilde{w}_{i}=\frac{\sqrt{w_{i}}}{\tilde{d}_{i}}$ for $i=2 N+1, \ldots, 3 N$, if $^{2}\left|\tilde{d}_{i}\right| \leq \rho_{i}$

$$
\begin{gathered}
\boldsymbol{A}_{1}=\left[\begin{array}{cc}
\vdots & \vdots \\
2 \boldsymbol{a}_{i}^{T} & -1 \\
\vdots & \vdots \\
2 \boldsymbol{a}_{i}^{T} & -1 \\
\vdots & \vdots \\
-2 \boldsymbol{a}_{i}^{T}, \text { if }\left|\tilde{d}_{i}\right| \leq \rho_{i} & 1 \\
\vdots & \vdots
\end{array}\right], \boldsymbol{p}_{1}=\left[\begin{array}{c}
\left\|\boldsymbol{a}_{i}\right\|^{2}-\left(\tilde{d}_{i}+\rho_{i}\right)^{2} \\
\vdots \\
\left\|\boldsymbol{a}_{i}\right\|^{2}-\left(\tilde{d}_{i}-\rho_{i}\right)^{2} \\
\vdots \\
-\left\|\boldsymbol{a}_{i}\right\|^{2}, \text { if }\left|\tilde{d}_{i}\right| \leq \rho_{i} \\
\vdots \\
\boldsymbol{I}_{k} \\
\mathbf{0}_{k \times 1} \\
0
\end{array}\right], \boldsymbol{g}=\left[\begin{array}{c}
\mathbf{0}_{k \times 1} \\
-1 / 2
\end{array}\right] .
\end{gathered}
$$

Both the objective function and the constraint in (17) are quadratic. This type of problem is known as GTRS [17], and its exact solution is readily obtain by a bisection procedure.

The estimate of $\boldsymbol{x}, \hat{\boldsymbol{x}}$, obtained from (17), can then be exploited to find an ML estimate of the target's CS, $\widehat{\omega}_{0}$, (i.e., we get $\left.\widehat{\delta}_{0}=1-\widehat{\omega}_{0}\right)$ as

$$
\widehat{\omega}_{0}=\frac{\sum_{i=1}^{N} \tilde{d}_{i}\left(\left\|\hat{\boldsymbol{x}}-\hat{\boldsymbol{a}_{i}}\right\|+\kappa\right)^{2}}{\sum_{i=1}^{N}\left(\left\|\hat{\boldsymbol{x}}-\hat{\boldsymbol{a}_{i}}\right\|+\kappa\right)^{2}}
$$

where $\kappa$ is defined in Algorithm 1. Then, $\widehat{\omega}_{0}$ is used to further enhance the localization accuracy, i.e., from (6), we write

$$
d_{i}-\frac{l_{i}+u_{i}}{4}-\widehat{\delta}_{0} d_{i} \approx\left\|\boldsymbol{x}-\boldsymbol{a}_{i}\right\|+\frac{d_{T_{i}}}{2}-\frac{l_{i}+u_{i}}{4}+\frac{n_{i}}{2} .
$$

Define $\quad \bar{d}_{i}=d_{i}-\frac{l_{i}+u_{i}}{4}-\widehat{\delta}_{0} d_{i} \quad$ and $\quad \bar{e}_{i}=d_{T_{i}} / 2-\left(l_{i}+\right.$ $\left.u_{i}\right) / 4$ and follow similar reasoning as described above to get

$$
\underset{\boldsymbol{y}=\left[\boldsymbol{x}^{T},\|\boldsymbol{x}\|^{2}\right]^{T}}{\operatorname{minimize}}\left\{\left\|\boldsymbol{W}_{2}\left(\boldsymbol{A}_{2} \boldsymbol{y}-\boldsymbol{p}_{2}\right)\right\|^{2}: \boldsymbol{y}^{T} \boldsymbol{D} \boldsymbol{y}+2 \boldsymbol{g}^{T} \boldsymbol{y}=0\right\}
$$

\footnotetext{
${ }^{2}$ Note that the minimum length of $\widetilde{\boldsymbol{w}}$ is $2 N$ if the condition is not met for any
} $i$, and its maximum length can be $3 N$.
TABLE I

SUMMARY OF THE CONSIDERED ALGORITHMS

\begin{tabular}{llc}
\hline \hline Algorithm & \multicolumn{1}{c}{ Description } & Complexity \\
\hline RSR-WLS & The proposed method in Algorithm 1 & $3 \times \mathcal{O}(K N)$ \\
LLS & The LLS method in [11] & $\mathcal{O}(N)$ \\
EDM & The EDM method in [12] & $\mathcal{O}\left(N^{4.5}\right)$ \\
CCCP-SOCP & The SOCP method in [15] & $\mathcal{O}\left(N^{3.5}\right)$ \\
SDP & The SDP method in [14] & $\mathcal{O}\left(N^{4.5}\right)$ \\
R-SOCP & The R-SOCP method in [16] & $\mathcal{O}\left(N^{3.5}\right)$ \\
EGTR & The extended GTR method in [15] & $\mathcal{O}(K N)$ \\
\hline \hline
\end{tabular}

where $\boldsymbol{W}_{2}=\boldsymbol{W}_{1}$, only with a slightly different condition: $\left|\bar{d}_{i}\right| \leq \bar{\rho}_{i}$, for $i=2 N+1, \ldots, 3 N$, with $\bar{\rho}_{i} \triangleq\left(u_{i}-l_{i}\right) / 4$, and

$$
\boldsymbol{A}_{2}=\left[\begin{array}{cc}
\vdots & \vdots \\
2 \boldsymbol{a}_{i}^{T} & -1 \\
\vdots & \vdots \\
2 \boldsymbol{a}_{i}^{T} & -1 \\
\vdots & \vdots \\
-2 \boldsymbol{a}_{i}^{T}, \text { if }\left|\bar{d}_{i}\right| \leq \bar{\rho}_{i} & 1 \\
\vdots & \vdots
\end{array}\right], \boldsymbol{p}_{2}=\left[\begin{array}{c}
\vdots \\
\left\|\boldsymbol{a}_{i}\right\|^{2}-\left(\bar{d}_{i}+\bar{\rho}_{i}\right)^{2} \\
\vdots \\
\left\|\boldsymbol{a}_{i}\right\|^{2}-\left(\bar{d}_{i}-\bar{\rho}_{i}\right)^{2} \\
\vdots \\
-\left\|\boldsymbol{a}_{i}\right\|^{2}, \text { if }\left|\bar{d}_{i}\right| \leq \bar{\rho}_{i} \\
\vdots
\end{array}\right]
$$

Now, we can use $\hat{x}$ and $\widehat{\omega}_{0}$ to find an ML estimate of the mean TAT $d_{T}, \widehat{d}_{T}$, as

$$
\widehat{d}_{T}=\frac{\sum_{i=1}^{N} 2 d_{i} / \widehat{\omega}_{0}-2\left\|\hat{\boldsymbol{x}}-\boldsymbol{a}_{i}\right\|}{N}
$$

which together with $\hat{\boldsymbol{x}}$ is used to update $\widehat{\omega}_{0}$ according to (18).

Finally, we exploit $\widehat{d}_{T}$ and $\widehat{\omega}_{0}$ to enhance the localization accuracy, i.e., from (2), for sufficiently small noise, we obtain

$$
\underset{\boldsymbol{x}}{\operatorname{minimize}} \sum_{i=1}^{N} w_{i}\left(\left\|\boldsymbol{x}-\boldsymbol{a}_{i}\right\|^{2}-r_{i}^{2}\right)^{2}
$$

where $r_{i}=d_{i} / \widehat{\omega}_{0}-\widehat{d}_{T} / 2$. Writing (22) in vector form yields

$$
\underset{\boldsymbol{y}=\left[\boldsymbol{x}^{T},\|\boldsymbol{x}\|^{2}\right]^{T}}{\operatorname{minimize}}\left\{\|\boldsymbol{W}(\boldsymbol{A} \boldsymbol{y}-\boldsymbol{p})\|^{2}: \boldsymbol{y}^{T} \boldsymbol{D} \boldsymbol{y}+2 \boldsymbol{g}^{T} \boldsymbol{y}=0\right\}
$$

where $\boldsymbol{W}=\operatorname{diag}\{\boldsymbol{w}\}, \boldsymbol{w}=\left[w_{i}\right]^{T}$, for $i=1, \ldots, N$

$$
\boldsymbol{A}=\left[\begin{array}{cc}
-2 \boldsymbol{a}_{1}^{T} & 1 \\
\vdots & \vdots \\
2 \boldsymbol{a}_{N}^{T} & 1
\end{array}\right], \boldsymbol{p}=\left[\begin{array}{c}
r_{1}^{2}-\left\|\boldsymbol{a}_{1}\right\|^{2} \\
\vdots \\
r_{N}^{2}-\left\|\boldsymbol{a}_{N}\right\|^{2}
\end{array}\right]
$$

Summary of the proposed alternating minimization algorithm, ${ }^{3}$ called "R-SR-WLS," is given in Algorithm 1. Note that at lines $3-4,7-8$, and $10-11$, we use knowledge about the ranges of the TAT and the CS [16], i.e., we do not let the respective estimates to be outside of the predefined interval.

\section{COMPLEXITY ANALYSIS}

Given $K$ as the maximum number of steps in the bisection procedure, Table I summarizes the computational complexities of the considered algorithms. It shows that the complexities of the R-SR-WLS, LLS, and EGTR are linear in $N$, while the complexity of other existing methods is significantly higher.

${ }^{3}$ Notice that one could continue iterating the steps designated by lines $6-12$ until a certain criteria is met. However, no gain was found in doing so. 


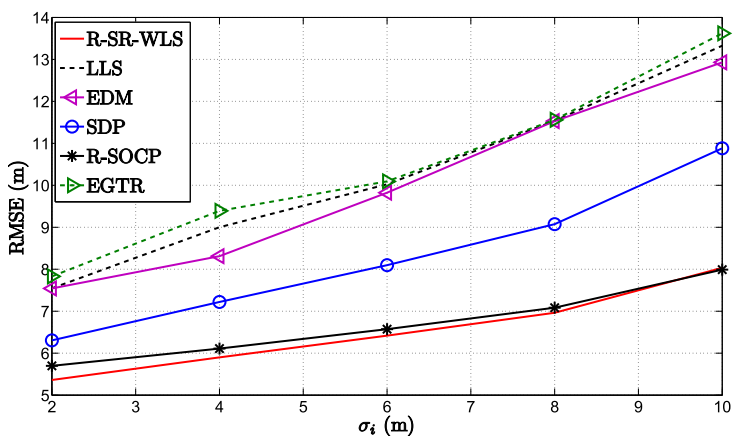

Fig. 1. RMSE versus $\sigma_{i}(\mathrm{~m})$ comparison, when $N=8, T_{0}=0.5 \mathrm{~ms}$, $\delta_{\max }=10^{-4}, B=0.5 \mathrm{~km}, M_{c}=3000$.

\section{Performance Results}

Here, the performance of the new algorithm is assessed through computer simulations and compared with the existing ones. $^{4,5,6}$ The measurements were generated by using (2). Eight anchors were fixed at $[0.4,0.4]^{T},[0.4,-0.4]^{T},[-0.4,0.4]^{T}$, $[-0.4,-0.4]^{T},[0.4,0]^{T},[0,0.4]^{T},[-0.4,0]^{T},[0,-0.4]^{T} \mathrm{~km}$, respectively, and the target was deployed randomly inside a $[-B, B] \times[-B, B] \mathrm{km}^{2}$ region in each Monte Carlo, $M_{c}$, run. The $\mathrm{CS}$ is set to $\omega_{i}=1+\delta_{i}$, for $i=0,1, \ldots, N$, where $\delta_{i}$ is drawn randomly from a uniform distribution on an interval $\left[-\delta_{\max }, \delta_{\max }\right]$, i.e., $\delta_{i} \sim \mathcal{U}\left[-\delta_{\max }, \delta_{\max }\right]$, with $\delta_{\max }=10^{-4}$, which corresponds to 100 PPM. The ideal TAT is set to $T_{0}=0.5 \mathrm{~ms}$ [21], and $K=30$ and $c=3 \times 10^{8} \mathrm{~m} / \mathrm{s}$ is used. $T_{i}^{\text {low }}$ and $T_{i}^{\text {upp }}$ are estimated in the calibration stage. If the target and anchors are equipped with same chips, it is reasonable to set $\bar{\mu}_{i}=\tilde{\mu}_{i}$ and $\bar{\sigma}_{i}=\tilde{\sigma}_{i}$ [16]. Note that in the existing literature [16], [18], $T_{i}$ is modeled as

$$
T_{i}=T_{\text {proc }}+\bar{n}_{i}=\omega_{i} T_{0}+\bar{n}_{i}, \text { for } i=1, \ldots, N
$$

where $T_{\text {proc }}=\omega_{i} T_{0}$ is the actual processing time, $\omega_{i}$ is the unknown CS, and $\bar{n}_{i} \sim \mathcal{N}\left(\bar{\mu}_{i}, \bar{\sigma}_{i}^{2}\right)$ is a random (positive) delay at the $i$ th anchor. Based on (24), the bounds can be computed as $T_{i}^{\text {low }}=\left(1-\delta_{\max }\right) T_{0}+\bar{\mu}_{i}-2.5 \bar{\sigma}_{i}$ and $T_{i}^{\text {upp }}=$ $\left(1+\delta_{\max }\right) T_{0}+\bar{\mu}_{i}+2.5 \bar{\sigma}_{i}$, so that $T_{i} \in\left[T_{i}^{\text {low }}, T_{i}^{\text {upp }}\right]$ in more than $99.379 \%$. Here, we assume that all sensors are equipped with the same chips, and we set $c \bar{\sigma}_{i}=c \tilde{\sigma}_{i}=\sigma_{i}$ and $\bar{\mu}_{i}=\tilde{\mu}_{i}=$ $3 \sigma_{i} / c, \forall i$. The performance metric used is the root mean square error (RMSE), defined as RMSE $=\sqrt{\sum_{i=1}^{M_{c}} \frac{\left\|\boldsymbol{x}_{i}-\widehat{\boldsymbol{x}}_{i}\right\|^{2}}{M_{c}}}$, where $\widehat{\boldsymbol{x}}_{i}$ denotes the estimate of the true target location, $\boldsymbol{x}_{i}$, in the $i$ th $M_{c}$ run.

Fig. 1 illustrates the RMSE versus $\sigma_{i}(\mathrm{~m})$ comparison. It can be seen from the figure that, not only that the new algorithm performs much better than the existing linear estimators, it matches or even outperforms the existing, considerably more complex ones as well. This can be explained to some extent by the fact that, we use an iterative approach and that after approximating the ML estimator by another nonconvex estimator, e.g. (16), we tackle it directly. In sharp contrast to the proposed approach, the existing convex-based methods further apply convex relaxation techniques to their derived approximations of the ML estimator,

\footnotetext{
${ }^{4}$ In order to make the comparison fair, the known ranges of the TATs were included as constraints in the EDM and the SDP methods.

${ }^{5}$ The EDM, the SDP, and the R-SOCP were implemented by using MATLAB package CVX [20], with SDPT3 solver.

${ }^{6}$ Note that, the CCP-SOCP [15] is omitted here, since it has exhibited poor performance in the considered scenarios [16].
}

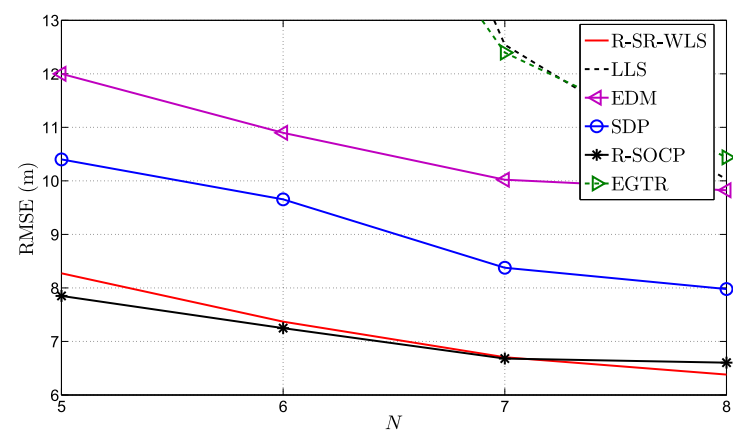

Fig. 2. RMSE versus $N$ comparison, when $\sigma_{i}=6 \mathrm{~m}, T_{0}=0.5 \mathrm{~ms}, \delta_{\max }=$ $10^{-4}, B=0.5 \mathrm{~km}, M_{c}=3000$.

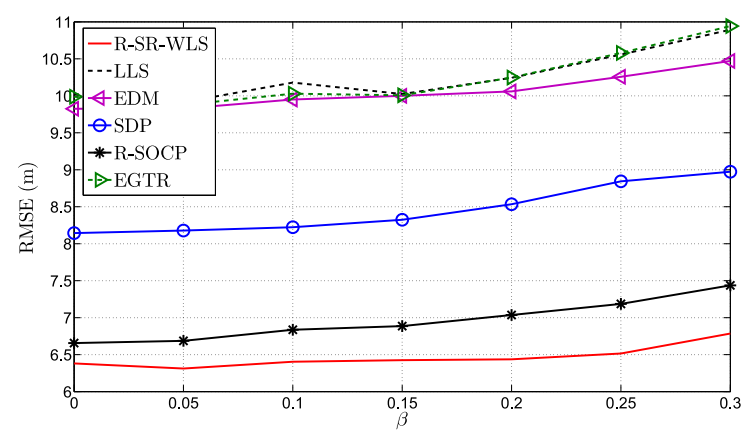

Fig. 3. RMSE versus $\beta$ comparison, when $N=8, \sigma_{i}=6 \mathrm{~m}, T_{0}=0.5 \mathrm{~ms}$, $\delta_{\max }=10^{-4}, B=0.5 \mathrm{~km}, M_{c}=3000$.

which expand the set of all possible solutions (the global optimum of such methods does not necessarily correspond (closely) to the global optimum of the original problem).

Fig. 2 illustrates the RMSE versus the first $N$ anchors comparison. This figure shows that the performance of all algorithms improves as $N$ increases, as expected. It also shows that the proposed algorithm matches the performance of the R-SOCP and outperforms significantly all other ones.

The assumption that the bounds on the TAT are perfectly known might not stand in practice. Hence, we examine the performance of all algorithms for a more practical scenario. We assume that imperfect estimates of $\tilde{\mu}_{i}$ and $\tilde{\sigma}_{i}$ are given as $\hat{\mu}_{i}=\tilde{\mu}_{i}\left(1+\varrho_{1}\right)$ and $\hat{\sigma}_{i}=\tilde{\sigma}_{i}\left(1+\varrho_{2}\right)$, respectively, with $\varrho_{1}, \varrho_{2} \sim \mathcal{U}[-\beta, \beta]$ and we present the results for the RMSE versus $\beta$ comparison in Fig. 3. It can be seen that the new algorithm ${ }^{7}$ is the most robust to these imperfections, and that it outperforms the existing ones for all range of $\beta$.

\section{CONCLUSION}

This letter addressed the problem of target localization using TW-ToA measurements with clock imperfections. A robust algorithm with linear computational complexity in $N$, whose solution is obtained exactly by merely a bisection procedure was proposed. Although the new algorithm requires knowledge about the ranges of the TAT, the simulation results showed that its performance is exceptional even when this knowledge is not perfectly available, and that it matches the performance of the SoA with significantly lower computational complexity.

\footnotetext{
${ }^{7}$ In our simulations, a significant improvement within each iteration of the new algorithm was observed in all scenarios. Nevertheless, for the sake of clarity of the figures, these results are omitted here.
} 


\section{REFERENCES}

[1] S. Tomic, M. Beko, and R. Dinis, "Distributed RSS-based localization in wireless sensor networks based on second-order cone programming," Sensors, vol. 14, no. 10, pp. 18410-18432, Oct. 2014.

[2] S. Tomic, M. Beko, and R. Dinis, "RSS-based localization in wireless sensor networks using convex relaxation: Noncooperative and cooperative schemes," IEEE Trans. Veh. Technol., vol. 64, no. 5, pp. 2037-2050, May 2015.

[3] S. Tomic, M. Beko, and R. Dinis, "3-D target localization in wireless sensor network using RSS and AoA measurement," IEEE Trans. Veh. Technol., vol. 66, no. 4, pp. 3197-3210, Apr. 2017.

[4] S. Tomic, M. Beko, and R. Dinis, "Distributed RSS-AoA based localization with unknown transmit powers," IEEE Wireless Commun. Lett., vol. 5, no. 4, pp. 392-395, Aug. 2016.

[5] S. Tomic, M. Beko, R. Dinis, and P. Montezuma, "A closed-form solution for RSS/AoA target localization by spherical coordinates conversion," IEEE Wireless Commun. Lett., vol. 5, no. 6, pp. 680-683, Dec. 2016.

[6] S. Tomic, M. Beko, R. Dinis, and P. Montezuma, "Distributed algorithm for target localization in wireless sensor networks using RSS and AoA measurements," Pervasive Mobile Comput., vol. 37, pp. 63-77, Oct. 2016.

[7] S. Tomic, M. Beko, R. Dinis, and P. Montezuma, "A robust bisection-based estimator for TOA-based target localization in NLOS environments," IEEE Commun. Lett., vol. 21, no. 11, pp. 2488-2491, Nov. 2017.

[8] S. Tomic and M. Beko, "A bisection-based approach for exact target localization in NLOS environments," Signal Process., vol. 143, pp. 328335, Feb. 2018.

[9] A. A. D’Amico, L. Taponecco, U. Mengali, "Ultra-wideband TOA estimation in the presence of clock frequency offset," IEEE Trans. Wireless Commun., vol. 126, no. 4, pp. 1606-1616, Apr. 2013.

[10] O. Bialer, D. Raphaeli, A. J. Weiss, "Two-way range estimation utilizing uplink and downlink channels dependency," IEEE Trans. Signal Process., vol. 62, no. 7, pp. 1619-1633, Apr. 2014
[11] M. R. Gholami, S. Gezici, and E. G. Ström, "Range based sensor node localization in the presence of unknown clock skews," in Proc. IEEE Int Conf. Acoust., Speech Signal Process., Vancouver, BC, Canada, May 2013, pp. 4046-4050.

[12] P. Oguz-Ekim, J. P. Gomes, P. Oliveira, M. R. Gholami, and E. G. Ström, "TW-TOA based cooperative sensor network localization with unknown turn-around time," in Proc. IEEE Int. Conf. Acoust., Speech Signal Process., Vancouver, BC, Canada, May 2013, pp. 6416-6420.

[13] M. R. Gholami, S. Gezici, and E. G. Ström, "TDOA based positioning in the presence of unknown clock skew," IEEE Trans. Commun., vol. 61 , no. 6, pp. 2522-2534, Mar. 2013.

[14] R. M. Vaghefi and R. M. Buehrer, "Asynchronous time-of-arrival-based source localization," in Proc. IEEE Int. Conf. Acoust., Speech Signal Process., Vancouver, BC, Canada, May 2013, pp. 6416-6420.

[15] M. R. Gholami, S. Gezici, and E. G. Ström, "TW-TOA based positioning in the presence of clock imperfections," Digit. Signal Process., vol. 59, pp. 19-30, Dec. 2016

[16] S. Gao, S. Zhang, G. Wang, and Y. Li, "Robust second-order cone relaxation for TW-TOA-based localization with clock imperfection," IEEE Signal Process. Lett., vol. 23, no. 8, pp. 1047-1051, Aug. 2016.

[17] A. Beck, P. Stoica, and J. Li, "Exact and approximate solutions of source localization problems," IEEE Trans. Signal Process., vol. 56, no. 5, pp. 1770-1778, May 2008.

[18] A. I. Baba, "Calibrating time of flight in two way ranging," in Proc. IEEE 17th Int. Conf. Mobile Ad-hoc Sensor Netw., Beijing, China, Dec. 2011 pp. 393-397.

[19] S. M. Kay., Fundamentals of Statistical Signal Processing: Estimation Theory. Upper Saddle River, NJ, USA: Prentice-Hall, 1993.

[20] M. Grant and S. Boyd, "CVX: Matlab software for disciplined convex programming," Version 1.21. [Online]. Available: http://cvxr.com/cvx. Accessed on Apr. 15, 2010.

[21] Annex D1: Location Topics, IEEE Standard 802.15.4a, Aug. 2007. 